\title{
Relationship Between Visual Estimates of Fusarium Head Blight Intensity and Deoxynivalenol Accumulation in Harvested Wheat Grain: A Meta-Analysis
}

\author{
P. A. Paul, P. E. Lipps, and L. V. Madden
}

Department of Plant Pathology, The Ohio State University, Ohio Agricultural Research and Development Center, Wooster 44691. Accepted for publication 10 July 2005.

\begin{abstract}
Paul, P. A., Lipps, P. E., and Madden, L. V. 2005. Relationship between visual estimates of Fusarium head blight intensity and deoxynivalenol accumulation in harvested wheat grain: A meta-analysis. Phytopathology 95:1225-1236.

The association between Fusarium head blight (FHB) intensity and deoxynivalenol (DON) accumulation in harvested grain is not fully understood. A quantitative review of research findings was performed to determine if there was a consistent and significant relationship between measures of Fusarium head blight intensity and DON in harvested wheat grain. Results from published and unpublished studies reporting correlations between DON and Fusarium head blight "index" (IND; field or plot-level disease severity), incidence (INC), diseased-head severity (DHS), and Fusarium-damaged kernels (FDK) were analyzed using metaanalysis to determine the overall magnitude, significance, and precision

correlation coefficients for all relationships between DON and disease intensity were significantly different from zero $(P<0.001)$. Based on the analysis of Fisher-transformed $r$ values ( $z_{r}$ values), FDK had the strongest relationship with DON, with a mean $r$ of 0.73 , followed by IND $(r=$ $0.62)$, DHS $(r=0.53)$, and INC $(r=0.52)$. The mean difference between pairs of transformed $z_{r}$ values $\left(\bar{z}_{d}\right)$ was significantly different from zero for all pairwise comparisons, except the comparison between INC and DHS. Transformed correlations were significantly affected by wheat type (spring versus winter wheat), study type (fungicide versus genotype trials), and study location (U.S. spring- and winter-wheat-growing regions, and other wheat-growing regions). The strongest correlations were observed in studies with spring wheat cultivars, in fungicide trials, and in studies conducted in U.S. spring-wheat-growing regions. There were minor effects of magnitude of disease intensity (and indirectly, environment) on the transformed correlations.
\end{abstract} of these associations. A total of 163 studies was analyzed, with estimated correlation coefficients $(r)$ between -0.58 and 0.99 . More than $65 \%$ of all $r$ values were $>0.50$, whereas less that $7 \%$ were $<0$. The overall mean
Additional keywords: effect size models, Fusarium graminearum, Gibberella zeae, wheat scab.
In North America, Fusarium head blight of wheat (Triticum aestivum L.) is caused predominantly by Fusarium graminearum Schwabe (teleomorph: Gibberella zeae). Very few plant diseases have had as great an impact on every sector of an industry as Fusarium head blight has had on the entire wheat industry in North America. Wheat growers, millers, bakers, and consumers of wheat products all may be affected by this disease (52). This is largely due to the fact that in addition to yield losses associated with reduced kernel size and weight, reduced seed germination, and seedling blight $(3,29), F$. graminearum also produces mycotoxins that may accumulate to unacceptable levels (44) in harvested grain, making the grain and their by-products unfit for commercialization and consumption. The two most common mycotoxins in wheat are deoxynivalenol (DON), commonly referred to as vomitoxin, and nivalenol (NIV) (28).

DON and NIV belong to a group of structurally similar fungal metabolites called trichothecenes. Compared with other members of this group, DON has a relatively low acute toxicity (44). In spite of this, it still is of major concern because it is a very common contaminant of grain and poses a serious health threat to

Corresponding author: L. V. Madden; E-mail address: madden.1@ osu.edu

The $\boldsymbol{e}$-Xtra logo stands for "electronic extra" and indicates that the online version contains supplemental material not included in the print edition. The online supplement contains a table with information regarding each individual study used in the meta-analysis.

DOI: 10.1094/PHYTO-95-1225

(C) 2005 The American Phytopathological Society humans and livestock $(44,46)$. DON-producing strains of $F$. graminearum (chemotype I) can be found worldwide, while NIVproducing strains (chemotype II) are restricted to parts of Asia and Europe (35). In the pathogenesis of Fusarium head blight, DON is considered a virulence factor $(5,31,39,40)$. The results of inoculation studies showed that although trichothecene-deficient mutants of $F$. graminearum were capable of inciting Fusarium head blight, disease severity was generally greater on spikes inoculated with wild-type (toxin-producing) strains than on those inoculated with mutant (non-toxin-producing) strains $(5,39)$. Desjardins et al. (5) and Proctor et al. (40) reported that when trichothecene-non-producing mutants were reverted (regaining the ability to produce toxins) wild-type virulence was restored. The level of trichothecenes produced by the reverted strains was comparable with that produced by the wild-type strains.

The role of DON in the development of Fusarium head blight and reports of positive relationships between fungal biomass and DON content of grain $(7,47,51)$ suggest that DON accumulation may be related to the level of Fusarium head blight damage. Varying degrees of association between Fusarium head blight intensity and DON accumulation in harvested grain have been reported in the literature, including situations with high positive correlations, low significant correlations, and negative correlations, as well as correlations close to zero $(1,2,4,7,12,13,24,25,27,31-34,45,51,54)$. Several questions regarding this association still remain unanswered. Is there an overall relationship between DON and some measure of Fusarium head blight intensity? If so, which measure of Fusarium head blight intensity has the strongest relationship with DON, and how significant and consistent is this 
relationship across studies? Is the relationship influenced by disease intensity level, DON level, or wheat type (winter wheat versus spring wheat)? Can disease intensity be used as a reliable indicator of DON levels in harvested grain?

Glass (10) proposed the use of meta-analysis as an objective approach for integrating and interpreting results and drawing conclusions from multiple studies. Meta-analysis is the statistical analysis of the results of statistical analyses from independent studies and provides a quantitative synthesis of research findings $(10,43)$. In meta-analysis, some measure of magnitude of treatment effect or association between variables (called effect size) is gathered from the results of individual studies, converted to a common metric, and analyzed to determine the magnitude, significance, heterogeneity, and precision of the mean effect size across studies (8). Relative to the more commonly used qualitative literature review, meta-analysis is regarded by many as a less biased summary of research findings $(10,43,50)$. This is partly because an objective set of rules is followed for inclusion of individual study results in the analysis, for quantifying the effect size, and for summarizing results from a population of studies. In addition, qualitative reviews often rely solely on a tally of the number of studies with significant results (i.e., those with $P<0.05$ ) to determine if a given treatment has an overall effect on an outcome or if an association is significant overall. Hedges and Olkin (16) showed how such methods can lead to erroneous conclusions. For example, if the sample size is large enough, even effects of marginal importance may achieve statistical significance $(11,50)$, leading to a distorted view of the overall effect. Very small $P$ values (highly significant results) in individual studies do not necessarily imply large overall effects, and very large $P$ values in individual studies do not necessarily imply small overall effects. As an alternative to significance testing, an estimate of the magnitude of treatment effect (effect size estimate) along with its confidence interval could be used to determine whether an effect is large enough to warrant consideration (50). Meta-analysis provides estimates of effect size and confidence intervals, and the sample size of each individual study is factored into the computing of the overall magnitude of these estimates. Moreover, effect size can be formally related to other variables that characterize the studies being used. Meta-analysis is widely used in the social sciences and medicine and has been advocated for plant pathology (41).

Understanding the relationship between Fusarium head blight intensity and DON accumulation could contribute to ongoing efforts to predict DON. Along with weather variables currently being employed in DON forecasting (19), disease intensity could potentially be used in prediction models as an indicator of DON accumulation. In addition, visual estimates of disease could also serve as indirect measures of DON to screen for genotypes with low DON accumulation. Direct quantification of DON is time consuming and expensive. With the objective of answering the aforementioned questions regarding relationships between disease intensity and DON, we used meta-analysis to conduct a quantitative synthesis of research findings on the relationships between visual estimates of Fusarium head blight intensity and DON accumulation in harvested grain. Both published and unpublished results were used in this analysis.

\section{MATERIALS AND METHODS}

Database for meta-analysis. To conduct a meta-analysis, it is first necessary to determine an effect size of interest and then decide how to collect the data to analyze this effect. For the purpose of analyzing research findings on the relationship between visual estimates of Fusarium head blight intensity and DON, a transformation of Pearson's product-moment correlation coefficient, $r$, was chosen as the effect size. This is one of the most commonly used statistics in meta-analysis, mainly because it is very easy to acquire or calculate from published and unpublished studies. Even when $r$ is not explicitly provided, it is straightforward to calculate using reported values of DON and disease intensity. This information was collected from studies published in peer-reviewed journals, nonreviewed articles, and unpublished studies. Relying solely on information from individual studies in the peer-reviewed literature to answer questions regarding the relationship between disease intensity and DON may lead to biased or even erroneous conclusions. Very often, significant findings are more likely to be published than nonsignificant findings (50). Rosenthal (42) used the term "file drawer problem" to refer to the conditional publishing of studies with significant results and the shelving or rejection of studies with nonsignificant results. Most of the unpublished data were acquired from the database of the U.S. Wheat and Barley Scab Initiative or from collaborators (D. Hershman, C. Sneller, M. McMullen, and G. Milus) compiling data from regional Fusarium head blight screening nurseries and Fusarium head blight fungicide trial plots. These studies were conducted according to standard protocols established by a panel of experts, and disease intensity and DON accumulation were evaluated in a similar manner in each study (see below).

In gathering data to conduct this analysis, a fixed set of criteria was used to determine whether or not a study would have been included in the analysis. First, the study had to provide at least one measure of Fusarium head blight intensity classified as one of the following: i) "index" (IND), defined as the average proportion of diseased spikelets per spike (sum of the proportion of visibly diseased spikelets per spike divided by the total number of spikes sampled, including those with zero severity); ii) incidence (INC), defined as the proportion of diseased spikes (number of spikes with nonzero severity divided by the total number of spikes sampled); iii) diseased-head severity (DHS), defined as the average proportion of diseased spikelets per diseased spike (sum of the proportion of diseased spikelets per diseased spike divided by the total number of diseased spikes sampled); or iv) Fusariumdamaged kernels (FDK), defined as the proportions of visually scabby kernels in a sample of harvested grain. Diseased-head severity is a form of "conditional severity" (30) since its determination is conditioned on the disease status of the spike. Disease index is sometimes referred to as "plot severity" (48) and is the more common measure of severity in epidemiology. With the exception of FDK, measurements of disease were made at approximately Feekes growth stage 11.2 (23). FDK was quantified after harvest. Second, DON had to be provided (in parts per million) as a measure of contamination of harvested grain (14) and not grain sampled during the growing season or spike chaff. Third, since not every pathogen capable of inciting Fusarium head blight produces DON, the study had to be conducted using DONproducing species of Fusarium (preferentially $F$. graminearum and F. culmorum) (in the case of artificial inoculation) or in an area where DON-producing species of Fusarium were predominant (in the case of natural inoculation).

A total of 36 published and 127 unpublished studies were selected for analysis. They represented 60 studies conducted to evaluate fungicide effects and 103 studies to evaluate genotype resistance over multiple years (1993 to 2004) and locations. Seventy studies were from U.S. winter-wheat-producing areas (MidAtlantic, southern, and Midwestern regions not including Iowa, Minnesota, and the Dakotas), 49 from U.S. spring-wheatproducing areas (Iowa, Minnesota, and the Dakotas), and the remaining 44 from Europe, Canada, or Africa. Fifty-eight of the studies had been conducted using spring wheat cultivars and 105 using winter wheat cultivars.

Meta-analysis of correlation coefficients. From each study, the Pearson product-moment correlation coefficient $(r)$ was obtained or calculated (using PROC CORR of SAS [Version 9.1]; SAS Institute, Cary, NC) for every combination of visual estimate of Fusarium head blight intensity and DON accumulation in har- 
vested grain: IND and DON, INC and DON, DHS and DON, and FDK and DON.

A separate meta-analysis was performed for each $r$ according to the Hedges-Olkin random-effects model $(8,17)$ using the meanes macro (26) in SAS. The analysis is considered a random-effects type because it is assumed that study has a random effect on effect size results which can be quantified by a between-study variance (explained below). Prior to the analysis, each $r$ value was transformed to correct for skewness in the sampling distribution (9) using Fisher's transformation

$$
z_{r_{i}}=\frac{1}{2} \log \left(\frac{1+r_{i}}{1-r_{i}}\right)
$$

in which $r_{i}$ and $Z_{r_{i}}$ refer to the correlation coefficient and its transformation for the $i$ th study, respectively. An additional advantage of $Z_{r_{i}}$ is that its asymptotic sampling variance is quantified as $v_{i}=$ $1 /\left(n_{i}-3\right)$, in which $n_{i}$ is the number of observations in the $i$ th study. Each $Z_{r_{i}}$ value was then weighted (an inverse function of its sampling variance) and used to estimate an overall mean $Z_{r_{i}}\left(\bar{Z}_{r}\right)$ as follows:

$$
\bar{z}_{r}=\frac{\sum_{i=1}^{k} w_{i} z_{r_{i}}}{\sum_{i=1}^{k} w_{i}}
$$

in which in the first stage of analysis, the weight is given as $w_{i}=$ $1 / v_{i}=n_{i}-3$, and $k$ is the total number of studies in the analysis. This first stage is equivalent to a fixed-effect model analysis, in which the effect size from each study is assumed to estimate the same population mean effect size (i.e., the effect size is constant across studies) and the only variability is within-study variation $\left(v_{i}\right)$. The $Z_{r_{i}}$ and $\bar{Z}_{r}$ values from the fixed-effect model were then used to calculate between-study variability for the random-effect model.

Chi-square test of homogeneity was performed for each correlation effect size $(8,17,26)$. The chi-square statistic ( $Q$ statistic), defined as the sum of the product of $w_{i}$ and the squared difference between $Z_{r_{i}}$ and $\bar{Z}_{r}$, was calculated as

$$
Q=\sum_{i=1}^{k}\left(n_{i}-3\right)\left(z_{r_{i}}-\bar{z}_{r}\right)^{2}
$$

A value of $Q$ larger than a theoretical chi-square value (with $k-$ 1 degrees of freedom) is an indication that a random-effects analysis is warranted. Based on $Q$, the between-study variance was calculated as

$$
\sigma^{2}=\frac{Q-(k-1)}{c}
$$

in which

$$
c=\sum_{i=1}^{k} w_{i}-\frac{\sum_{i=1}^{k} w_{i}^{2}}{\sum_{i=1}^{k} w_{i}}
$$

Use of both between- and within-study variation is considered the random-effects model analysis $(8,17)$. Thus, the variance of $Z_{r_{i}}$ for the $i$ th study was determined as $v_{i}^{*}=v_{i}+\sigma^{2}=\left(1 /\left(n_{i}-\right.\right.$ $3))+\sigma^{2}$. For the random-effects analysis, the mean effect size, $\bar{Z}_{r}$, was calculated with equation 2 using $w_{i}=1 / v_{\mathrm{i}}{ }^{*}=\left[\left(1 /\left(n_{i}-3\right)\right)\right.$ $\left.+\sigma^{2}\right]^{-1}=\left(v_{i}+\sigma^{2}\right)^{-1}$ as weights. The standard error of $\bar{Z}_{r}$ was estimated as

$$
\operatorname{se}\left(\bar{z}_{r}\right)=\sqrt{\frac{1}{\sum_{i=1}^{k} w_{i}}}
$$

and the $Z$ (standard normal) statistic as

$$
Z=\frac{\bar{z}_{r}}{\operatorname{se}\left(\bar{z}_{r}\right)}
$$

Values of $Z>1.96$ or $<-1.96$ indicate that the correlation was significantly different from zero at $P=0.05$. The upper and lower limits of the $95 \%$ confidence interval around $\bar{Z}_{r}$ were calculated as $\bar{Z}_{r} \pm 1.96 \times \operatorname{se}\left(\bar{z}_{r}\right)$.

Subsequent to the analyses, the $\bar{Z}_{r}$ values and confidence limits were back transformed using the following equation

$$
\bar{r}=\frac{e^{\left(2 \bar{z}_{r}\right)}-1}{e^{\left(2 \bar{z}_{r}\right)}+1}
$$

to obtain estimates of the untransformed mean correlation coefficients.

Comparing correlation coefficients. We next quantified the differences in the correlation coefficients between DON and the different measures of Fusarium head blight intensity. For instance, it would be interesting to know whether the correlation between Fusarium head blight incidence and DON $\left(r_{\text {INC }}\right)$ was significantly different from the correlation between Fusarium head blight index and DON $\left(r_{\text {IND }}\right)$ or whether the correlation between diseasedhead severity and DON $\left(r_{\text {DHS }}\right)$ was significantly different from the correlation between Fusarium-damaged kernels and DON $\left(r_{\text {FDK }}\right)$. For this purpose, the null hypothesis of no difference between pairs of correlation coefficients was tested.

All measurements of disease intensity were not made in most studies. Thus, for example, to determine whether the correlation of disease index and DON was significantly higher (lower) than the correlation of disease incidence with DON, subsets of the total data set were constructed that contained only those studies with the two disease intensity variables (and thus, two correlation coefficients) of interest. Because both disease intensity measurements were made on the same experimental units (e.g., plots), the calculated correlations were dependent. That is, the correlations were correlated with each other. A number of approaches for testing the null hypothesis of no difference between pairs of dependent correlations have been proposed $(6,18,49)$. Among these approaches, the method proposed by Dunn and Clark (6) stands out for its relative robustness to magnitude of predictor intercorrelation, high statistical power, and relatively low Type I error rate for large and normally distributed populations (18). The Dunn and Clark method was used to test the equality of pairs of $z_{r}$ values rather than the equality of pairs of $r$ values.

For a single data set, the method involves calculating the difference of pairs of Fisher-transformed correlations and the variance (or standard error) of the difference of $z_{r}$ values. Consider, for example, a single study and the correlations between DON and index $\left(r_{\text {IND }}\right)$ and DON and incidence $\left(r_{\text {INC }}\right)$. The difference in transformed $r$ is $z_{d}=z_{r(\mathrm{IND})}-z_{r(\mathrm{INC})}$, and the asymptotic variance of this variable is

$$
v_{d}=\frac{2\left(1-r_{\mathrm{IND}, \mathrm{INC}}\right)}{n-3}
$$

in which $r_{\text {IND,INC }}$ is the correlation between $r_{\text {IND }}$ and $r_{\text {INC }}$, which is a function of the correlations of both IND and INC with DON ( $r_{\mathrm{IND}}$ and $r_{\mathrm{INC}}$ ) and the correlation of IND and INC in the study (given by equation 8 in citation 6). When the two correlations are independent, $r_{\text {IND,INC }}=0$ and $v_{\mathrm{d}}=2 /(n-3)$.

The meta-analysis was conducted based on differences in $z_{r}$ values $\left(z_{d_{i}}\right)$ as effect sizes instead of on $z_{r_{i}}$ values. That is, $z_{r_{i}}$ was replaced by $z_{d_{i}}$ and $w_{i}$ by $1 / v_{d_{i}}$ (equation 9) to obtain an initial (fixed-effects) analysis. The test for homogeneity (equation 3 ) was then performed, and the calculated $Q$ statistic was used to obtain the between-studies variance of the correlation differences, $\sigma_{d}^{2}$. Then, to perform a random-effects analysis, a new weight was determined for the $i$ th study as $w_{i}=\left(v_{d_{i}}+\sigma_{d}^{2}\right)^{-1}$ and equations 
2, 6, and 7 were recalculated. With this substitution, equation 6 gives the estimated standard error of the difference of mean transformed correlations and equation 7 gives a test of the difference. In the latter case, a value of $Z$ in equation 7 greater than 1.96 or less than -1.96 indicates that the paired correlations are significantly different at $P=0.05$.

Influence of moderator variables. Given the fact that the studies represented data from multiple years and locations planted with different wheat genotypes, it seems unreasonable to assume that variability in effect size estimate across studies was merely due to sampling error (fixed-effect). Thus, in addition to the random-effects analysis described above, the effect sizes (transformed correlations) were analyzed using meta-analytic methods analogous to one-way analysis of variance (ANOVA) and regression to determine whether effect sizes were affected by one or more moderator variables (26). Computational details are given in Lipsey and Wilson (26).

Wheat type (spring or winter wheat), study location (U.S. spring- or winter-wheat-growing areas, or other wheat-growing areas), and study type (genotype evaluation or fungicide trial) were used as categorical (factor) moderator variables, whereas the mean, maximum, and difference between the maximum and mean of IND, INC, DHS, and DON for each study were used as continuous moderator variables to determine the potential influence of these variables on the strength of the correlations between DON and disease intensity (i.e., magnitude of $\bar{Z}_{r}$ ). The continuous and categorical moderator variables were considered fixed-effects terms, and study was considered a random-effect term; thus, the evaluation of moderator variables is considered a mixed-effect model analysis (in contrast to the purely randomeffects analysis when no moderator variables were considered). Mean disease intensity, maximum disease intensity, and mean and maximum DON level could be considered, in part, as indirect measures of environmental favorability for Fusarium head blight development and DON accumulation because one would expect disease intensity or DON concentration to increase, on average, with increasing favorable environmental conditions $(19,20,22)$.

For each categorical variable, the metaf macro (26) in SAS was used to obtain a separate mean $Z_{r_{i}}$ value for each category level (with associated confidence intervals and standard normal statistics). These values were compared across levels of each moderator variable using an approach analogous to one-way weighted ANOVA, where total variability $(Q)$ was partitioned into withinand between-group variability $\left(Q_{\mathrm{W}}\right.$ and $\left.Q_{\mathrm{B}}\right)$. As with the analyses without moderator variables, the weight was the inverse of the variance of each $Z_{r_{i}}$. The variance of each effect size was the sum of sampling variance and between-study variance, the latter being determined after accounting for the effects of the moderator variables. A significant effect of the moderator variable was based on a chi-square test of the between-group sum of squares (with degrees of freedom equal to the number of groups minus 1).

For the continuous moderator variables, the metareg macro (26) was used to fit weighed regression-type models relating effect size estimates $\left(Z_{r_{i}}\right)$ to moderator variables. As with the categorical moderator variables, the weight was the inverse of the variance of each $Z_{r_{i}}$ (with a variance equal to the sum of the sampling variance and the between-study variance, the latter being determined after adjusting for the effect of the moderator variable on the effect size).

For the mixed-effects analyses, estimation of the between-study variance, mean effect size, standard error of mean effect size, and regression parameters (slope and intercept) and their standard errors was done with the method of moments (26).

\section{RESULTS}

Correlation between DON and Fusarium head blight intensity. The association between Fusarium head blight intensity and
DON accumulation in harvested grain varied from one study to another and among the different measures of disease intensity within any given study. Some studies reported very strong positive correlations between DON and some measures of disease intensity, whereas for other studies and/or measures of disease, close to zero or negative correlations were reported (Fig. 1). The correlation between disease index and DON $\left(r_{\mathrm{IND}}\right)$ ranged from -0.44 to 0.99 , between disease incidence and DON $\left(r_{\text {INC }}\right)$ from -0.58 to 0.97 , between diseased-head severity and DON ( $\left.r_{\text {DHS }}\right)$ from -0.43 to 0.94 , and between Fusarium-damaged kernels and DON $\left(r_{\mathrm{FDK}}\right)$ from -0.47 to 0.98 (Fig. 1). Seventy percent of 158 studies reported $r_{\text {IND }}>0.50,60 \%$ of 119 reported $r_{\text {INC }}>0.50,55 \%$ of 109 reported $r_{\mathrm{DHS}}>0.50$, and $80 \%$ of 100 reported $r_{\mathrm{FDK}}>0.50$. Eight percent of the studies had negative $r_{\text {IND }}, 5.8 \%$ had negative $r_{\mathrm{INC}}, 11 \%$ had negative $r_{\mathrm{DHS}}$, and $2 \%$ had negative $r_{\mathrm{FDK}}$.

The distributions of $r$ across studies for all of the relationships between DON and disease intensity were negatively skewed (Fig. 1). This justified the use of a transformation-based meta-analytic approach as opposed to an approach based on untransformed $r$. For all relationships, the distributions of Fisher's transformation of $r\left(z_{r}\right)$ and the differences between pairs of $z_{r}\left(z_{d}\right)$ were symmetrical about their means and reasonably described by a normal distribution (Figs. 1 and 2). The relative positions of the distribution curves on the horizontal axes (Figs. 1B, D, F, and H and 2) clearly differed among some of the measures of intensity.

Meta-analysis of effect sizes. The null hypothesis of homogeneity was rejected for all effect sizes ( $z_{r}$ and $z_{d}$ values), suggesting that each individual study estimated a different populationeffect size rather than giving estimates of a single-effect size across all studies. This justifies the use of random-effects meta-analysis in which the variability in effect size estimates is greater than that which can be attributed to just random sampling error (26).

The overall mean effect size, $\bar{Z}_{r}$, varied from one measure of disease to another. For all relationships between DON and disease intensity, $\bar{z}_{r}$ was significantly different from zero based on the standard normal test $(P<0.001)$. FDK, quantified after harvest, had the strongest association with DON accumulation in harvested grain, with a $\bar{Z}_{r}$ value of 0.93 (Table 1). This corresponded to an overall mean back-transformed correlation coefficient $(\bar{r})$ across all studies of $\bar{r}_{\mathrm{FDK}}=0.73$. Among the measures of disease intensity quantified prior to harvest (at GS 11.2), IND had the highest $\bar{r}$ value across all studies $\left(\bar{r}_{\text {IND }}=0.62\right)$, followed by DHS $\left(\bar{r}_{\mathrm{DHS}}=0.53\right)$ and INC $\left(\bar{r}_{\mathrm{INC}}=0.52\right)$. The width of the $95 \%$ confidence interval around $\bar{r}_{\text {IND }}(0.09)$ was narrower than those around $\bar{r}_{\text {DHS }}(0.12)$ and $\bar{r}_{\text {INC }}(0.10)$ (Table 1$)$. The confidence intervals around $\bar{r}_{\mathrm{IND}}$ and $\bar{r}_{\mathrm{IND}}, \bar{r}_{\mathrm{IND}}$ and $\bar{r}_{\mathrm{FDK}}$, and $\bar{r}_{\mathrm{INC}}$ and $\bar{r}_{\mathrm{FDK}}$ did not overlap, suggesting a difference between each of these pairs. The confidence intervals around $\bar{r}_{\mathrm{INC}}$ and $\bar{r}_{\mathrm{DHS}}$ were almost identical, suggesting that $\bar{r}_{\mathrm{INC}}$ and $\bar{r}_{\mathrm{DHS}}$ were not different. Formal testing of equality of correlations (or more specifically, their Fisher transformations), however, must be based on pairwise differences between $z_{r}$ values for each study where the two measurements of interest were made.

The null hypothesis of no difference between pairs of $z_{r}$ was rejected for all but one comparison based on the standard normal test (Table 2). Except for the difference between $z_{r(\mathrm{INC})}$ and $z_{r(\mathrm{DHS})}$, the mean difference $\left(\bar{z}_{d}\right)$ was significantly different from zero $(P<0.001)$. The $95 \%$ confidence interval around the mean difference between $z_{r(\mathrm{INC})}$ and $z_{r(\mathrm{DHS})}$ included zero, hence the overall mean difference between these two values was considered to be zero, indicating that the correlation between DON and incidence was the same as the correlation between DON and diseased-head severity. For the other comparisons, the confidence intervals around $\bar{z}_{d}$ did not include zero (Table 2). The greatest mean difference (in absolute value) was between $z_{r(\mathrm{INC})}$ and $z_{r(\mathrm{FDK})}$. Based on the pairwise differences, FDK had the highest correlation overall with DON, followed by disease index, and then incidence and diseased-head severity. 
Influence of moderator variables. Study location, wheat type, and study type all had significant effects on the magnitude of $\bar{z}_{r}$ $(P<0.001)$. For all relationships between disease intensity and DON, studies conducted in U.S. spring-wheat-growing regions yielded significantly higher overall mean transformed and backtransformed correlation coefficients than studies conducted in U.S. winter-wheat-growing regions (Table 3). The overall mean effect sizes were from 0.21 to 0.46 higher for U.S. spring wheat
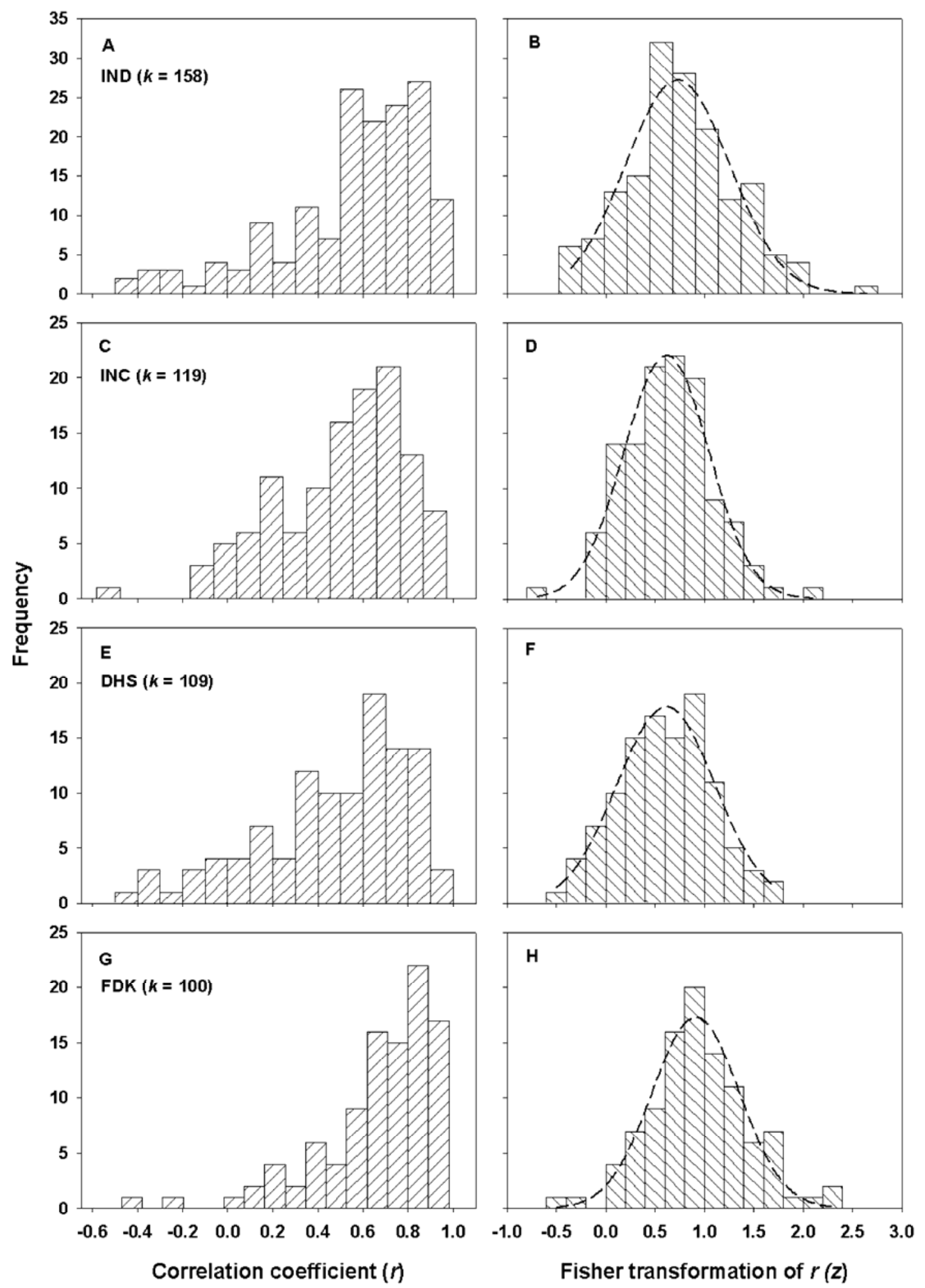

Fig. 1. Frequency distribution of Pearson's product-moment correlation coefficients $(r)(\mathbf{A}, \mathbf{C}, \mathbf{E}, \mathbf{G})$ and Fisher's $z$ transformations of $r(\mathbf{B}, \mathbf{D}, \mathbf{F}, \mathbf{H})$ for the relationships between deoxynivalenol (DON) accumulation in harvested wheat grain and Fusarium head blight "index" (IND = mean proportion of diseased spikelets per spike) $(\mathbf{A}, \mathbf{B})$, incidence $(\mathrm{INC}=$ proportion of diseased spikes) $(\mathbf{C}, \mathbf{D})$, diseased-head severity $(\mathrm{DHS}=$ mean proportion of diseased spikelets per diseased spike) (E, F), and Fusarium-damaged kernels (FDK = proportion of visibly scabby kernels) $(\mathbf{G}, \mathbf{H})$. The smooth curves in $\mathbf{B}, \mathbf{D}, \mathbf{F}$, and $\mathbf{H}$ represent the normal distribution function fitted to the data for each relationship. $k$ is the number of studies in which the indicated variable was measured. 
compared with U.S. winter-wheat-growing areas. In general, studies from outside the U.S. spring and winter wheat regions (Europe, Canada, and Africa) resulted in associations between measures of disease intensity and DON comparable with or stronger than that found for studies from U.S. winter wheat areas, and weaker than that found for studies from U.S. spring wheat areas. Within any given study location, disease index showed the strongest correlation with DON accumulation among measures of disease intensity quantified prior to harvest.

When wheat type was used as a moderator variable (regardless of study type or location), studies in which spring wheat cultivars were planted resulted in significantly higher correlation coefficients than studies planted with winter wheat cultivars $(P<0.05$, Table 4) for all but one relationship between disease intensity and DON. Only the relationship between FDK and DON was not significantly influenced by wheat type $(P>0.05$; Table 4$)$. When study type was considered, fungicide evaluation studies had sig- nificantly higher correlations between IND and DON and INC and DON than genotype evaluation studies $(P<0.05$; Table 5$)$. Study type did not have a significant effect on the magnitude of $\bar{z}_{r}$ for relationships between DHS and DON and FDK and DON. Within any given wheat type or study type, FDK had the highest overall $\bar{z}_{r}$ (and hence, $\bar{r}$ ) value. Among measures of disease quantified prior to harvest, disease index had the highest $\bar{z}_{r}$ value. In general, the strongest association between disease and DON occurred in studies where spring wheat cultivars were planted in fungicide evaluation plots, with overall $\bar{z}_{r(\mathrm{IND})}, \bar{z}_{r(\mathrm{INC})}, \bar{z}_{r(\mathrm{DHS})}$, and $\bar{z}_{r(\mathrm{FDK})}$ values of $1.086,1.001,1.008$, and 0.960 , respectively. The corresponding back-transformed correlation coefficients were $0.80,0.76,0.76$, and 0.74 .

For the analysis of the effects of continuous moderator variables on effect sizes, the mean, maximum, and difference between maximum and mean (roughly half the data range, labeled as range for short) of disease index, incidence, diseased-head severity, and
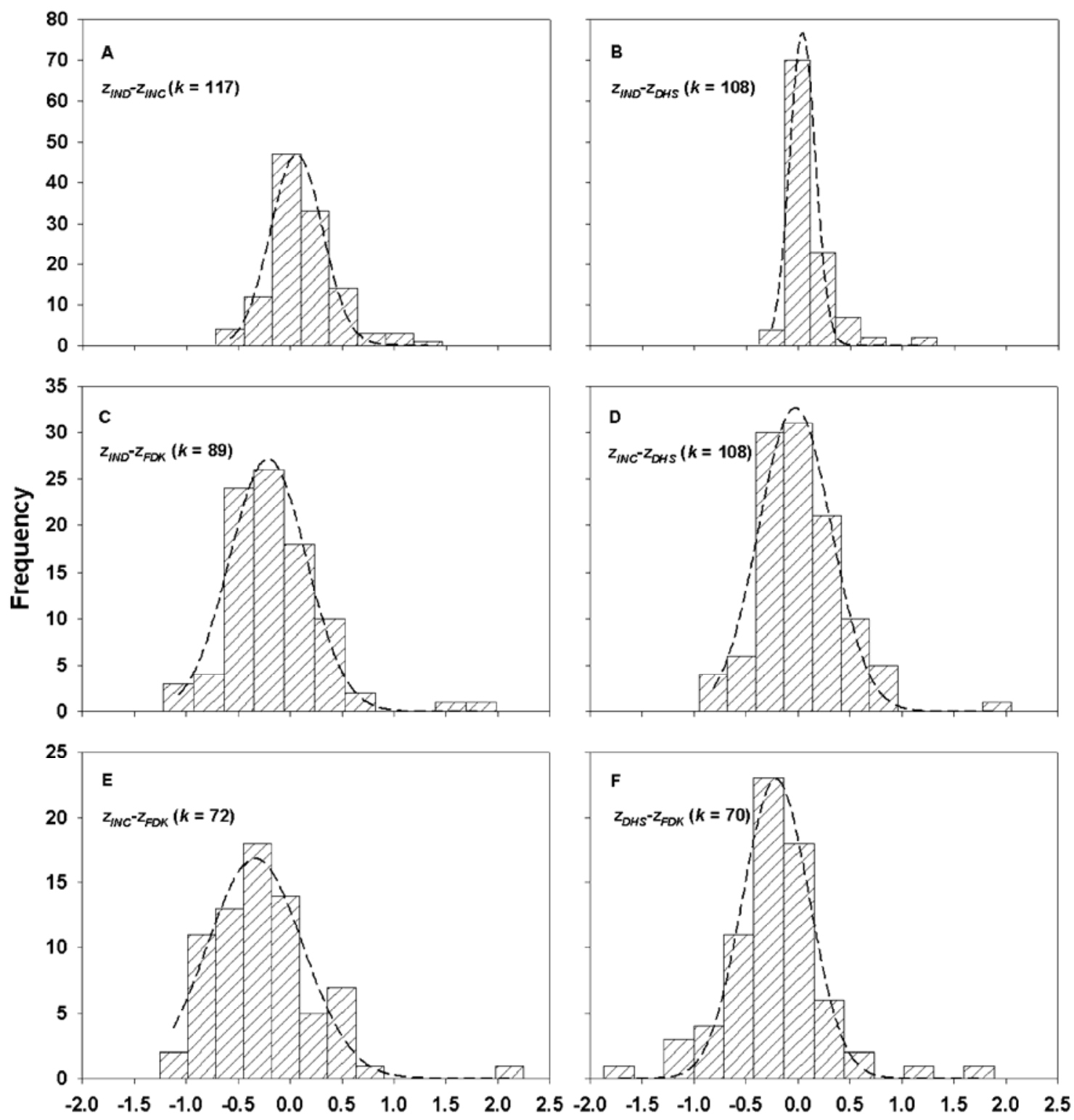

Fisher transformation of $r(z)$

Fig. 2. Frequency distribution of differences between pairs of Fisher's $z$ transformations of Pearson's product-moment correlation coefficients for relationships between deoxynivalenol (DON) accumulation in harvested wheat grain and Fusarium head blight "index" (IND = mean proportion of diseased spikelets per spike), incidence (INC $=$ proportion of diseased spikes), diseased-head severity (DHS = mean proportion of diseased spikelets per diseased spike), and Fusarium-damaged kernels (FDK $=$ proportion of visibly scabby kernels). The smooth curves represent the normal distribution function fitted the data for each relationship. $k$ is the number of studies in which both indicated disease variables were measured. 
DON explained only a very small proportion of the variability (Table 6). Even though the regression models and model parameters were statistically significant for some relationships between moderator variables and effect sizes, the highest proportion of the variability explained by any given model was only $9.1 \%$. Three- quarters of the regressions had $R^{2}$ values $<5 \%$. The slopes of the regression equations were either positive or negative. If one considers only those regression results with $P \leq 0.05$ (roughly, $R^{2}>3 \%$ ), one finds mostly a negative relationship (i.e., slope) between the effect size and moderator variable. For instance, there

TABLE 1. Transformed and back-transformed correlation coefficients and corresponding statistics for relationships between different measures of Fusarium head blight intensity and deoxynivalenol accumulation in harvested wheat grain

\begin{tabular}{|c|c|c|c|c|c|c|c|c|c|c|}
\hline \multirow[b]{2}{*}{ Relationship $^{\mathrm{a}}$} & \multirow[b]{2}{*}{$k^{\mathrm{b}}$} & \multicolumn{6}{|c|}{ Transformed correlations $^{\mathrm{c}}$} & \multicolumn{3}{|c|}{ Correlations $^{\mathrm{d}}$} \\
\hline & & $\bar{z}_{r}$ & $\operatorname{se}\left(\bar{z}_{r}\right)$ & $C I_{\mathrm{L}}$ & $C I_{\mathrm{U}}$ & $Z$ & $P$ & $\bar{r}$ & $C I_{\mathrm{L}}$ & $C I_{\mathrm{U}}$ \\
\hline IND vs. DON & 158 & 0.73 & 0.037 & 0.65 & 0.80 & 19.43 & $<0.001$ & 0.62 & 0.57 & 0.66 \\
\hline INC vs. DON & 119 & 0.58 & 0.036 & 0.51 & 0.65 & 16.26 & $<0.001$ & 0.52 & 0.47 & 0.57 \\
\hline FDK vs. DON & 100 & 0.93 & 0.047 & 0.84 & 1.03 & 19.74 & $<0.001$ & 0.73 & 0.69 & 0.77 \\
\hline
\end{tabular}

${ }^{a}$ DON = deoxynivalenol concentration in harvested grain (ppm); IND = Fusarium head blight index (mean proportion of diseased spikelets per spike); INC = Fusarium head blight incidence (proportion of diseased spikes); DHS = diseased-head severity ("conditional severity"; mean proportion of diseased spikelets per diseased spike); FDK = Fusarium-damaged kernels (proportion of visibly scabby kernels at harvest).

${ }^{\mathrm{b}}$ Total number of studies used in each analysis (based on the number of studies in which the indicated variable was measured).

c $\bar{z}_{r}=$ Mean Fisher's transformed correlation coefficient; se $\left(\bar{z}_{r}\right)=$ standard error of $\bar{z}_{r}$; lower $\left(C I_{\mathrm{L}}\right)$ and upper $\left(C I_{\mathrm{U}}\right)$ limits of the $95 \%$ confidence interval around $\bar{z}_{r}$; and $Z$ (standard normal) statistic from the meta-analysis of relationships between DON and measures of Fusarium head blight intensity; and $P=$ probability value (significance level).

${ }^{\mathrm{d}}$ Mean back-transformed correlation coefficients $(\bar{r})$ and lower $\left(C I_{\mathrm{L}}\right)$ and upper $\left(C I_{\mathrm{U}}\right)$ limits of the $95 \%$ confidence interval obtained through back-transformation of the corresponding transformed values.

TABLE 2. Pairwise differences between Fisher's transformed correlation coefficients and their corresponding statistics for relationships between different measures of Fusarium head blight intensity and deoxynivalenol accumulation in harvested wheat grain

\begin{tabular}{|c|c|c|c|c|c|c|c|}
\hline \multirow[b]{2}{*}{ Comparison $^{\mathrm{a}}$} & \multirow[b]{2}{*}{$k^{\mathrm{b}}$} & \multicolumn{6}{|c|}{ Statistics $^{\mathrm{c}}$} \\
\hline & & $\bar{z}_{d}$ & $\operatorname{se}\left(\bar{z}_{d}\right)$ & $C I_{\mathrm{L}}$ & $C I_{\mathrm{U}}$ & $Z$ & $P$ \\
\hline$z_{d}=z_{r(\mathrm{IND})}-z_{r(\mathrm{INC})}$ & 117 & 0.09 & 0.021 & 0.05 & 0.13 & 4.21 & $<0.001$ \\
\hline$z_{d}=z_{r(\mathrm{IND})}-z_{r(\mathrm{DHS})}$ & 108 & 0.03 & 0.008 & 0.01 & 0.04 & 3.75 & $<0.001$ \\
\hline$z_{d}=z_{r(\mathrm{INC})}-z_{r(\mathrm{DHS})}$ & 108 & -0.03 & 0.031 & -0.09 & 0.03 & -0.88 & 0.378 \\
\hline$z_{d}=z_{r(\mathrm{INC})}-z_{r(\mathrm{FDK})}$ & 71 & -0.28 & 0.047 & -0.38 & -0.19 & -6.02 & $<0.001$ \\
\hline$z_{d}=z_{r(\mathrm{DHS})}-z_{r(\mathrm{FDK})}$ & 70 & -0.20 & 0.040 & -0.28 & -0.12 & -5.06 & $<0.001$ \\
\hline
\end{tabular}

${ }^{a}$ Comparisons between differences of Fisher's transformed correlation coefficients $\left(z_{r}\right)$ for relationships between deoxynivalenol concentration in harvested grain (ppm) and Fusarium head blight index (IND = mean proportion of diseased spikelets per spike), incidence (INC = proportion of diseased spikes), diseased-head severity (DHS = mean proportion of diseased spikelets per diseased spikes), and Fusarium-damaged kernels (FDK = proportion of visibly scabby kernels).

${ }^{b}$ Total number of studies used in each analysis (based on the number of studies where both indicated disease variables were measured).

c $\bar{z}_{d}=$ Estimated mean of paired differences of transformed correlation coefficient; standard error (se) and lower $\left(C I_{\mathrm{L}}\right)$ and upper $\left(C I_{\mathrm{U}}\right)$ limits of the $95 \%$ confidence interval of $\bar{z}_{d} ; Z$ (standard normal) statistic from the analysis of the estimated difference; and $P=$ probability value (significance level).

TABLE 3. Fisher's transformed and back-transformed correlation coefficients and corresponding statistics for the effect of wheat-growing region on the relationship between different measures of Fusarium head blight intensity and deoxynivalenol accumulation in harvested wheat grain

\begin{tabular}{|c|c|c|c|c|c|c|c|c|c|c|}
\hline \multirow[b]{2}{*}{$\underline{\text { Relationship }}^{\mathrm{a}}$} & \multirow[b]{2}{*}{ Location $^{\mathrm{b}}$} & \multirow[b]{2}{*}{$k^{\mathrm{c}}$} & \multicolumn{5}{|c|}{ Transformed correlations $^{\mathrm{d}}$} & \multicolumn{3}{|c|}{ Correlations ${ }^{\mathrm{e}}$} \\
\hline & & & $\bar{z}_{r}$ & $\operatorname{se}\left(\bar{z}_{r}\right)$ & $C I_{\mathrm{L}}$ & $C I_{\mathrm{U}}$ & $P$ value & $\bar{r}$ & $C I_{\mathrm{L}}$ & $C I_{\mathrm{U}}$ \\
\hline \multirow{2}{*}{$\overline{\text { IND vs. DON }}$} & Winter & 66 & 0.54 & 0.055 & 0.43 & 0.65 & $<0.001$ & 0.49 & 0.41 & 0.57 \\
\hline & Spring & 47 & 0.91 & 0.068 & 0.78 & 1.05 & & 0.72 & 0.65 & 0.78 \\
\hline \multirow[t]{3}{*}{ INC vs. DON } & Winter & 59 & 0.51 & 0.049 & 0.42 & 0.61 & 0.034 & 0.47 & 0.40 & 0.54 \\
\hline & Spring & 39 & 0.72 & 0.063 & 0.59 & 0.84 & & 0.62 & 0.53 & 0.69 \\
\hline & Other & 21 & 0.54 & 0.088 & 0.37 & 0.71 & & 0.49 & 0.35 & 0.61 \\
\hline \multirow[t]{3}{*}{ FDK vs. DON } & Winter & 44 & 0.73 & 0.066 & 0.60 & 0.86 & $<0.001$ & 0.62 & 0.54 & 0.70 \\
\hline & Spring & 39 & 1.03 & 0.071 & 0.89 & 1.17 & & 0.77 & 0.71 & 0.82 \\
\hline & Other & 11 & 1.27 & 0.131 & 1.02 & 1.53 & & 0.85 & 0.77 & 0.91 \\
\hline
\end{tabular}

a DON = deoxynivalenol concentration in harvested grain $(\mathrm{ppm})$; IND = Fusarium head blight index (mean proportion of diseased spikelets per spike); INC = Fusarium head blight incidence (proportion of diseased spikes); DHS = diseased-head severity ("conditional severity"; mean proportion of diseased spikelets per diseased spike); FDK = Fusarium-damaged kernels (proportion of visibly scabby kernels).

${ }^{\mathrm{b}}$ Data obtained from studies conducted in U.S. winter wheat- and spring wheat-growing areas or from locations outside of the U.S. winter and spring wheat areas (other).

c Total number of studies used in each analysis (based in the number of studies in which the indicated variable was measured).

d $\bar{z}_{r}=$ Mean Fisher's transformed correlation coefficient; $\operatorname{se}\left(\bar{z}_{r}\right)=$ standard error of $\bar{z}_{r}$; lower $\left(C I_{\mathrm{L}}\right)$ and upper $\left(C I_{\mathrm{U}}\right)$ limits of the $95 \%$ confidence interval for $\bar{z}_{r} ; Z$ (standard normal) statistic from the analysis of relationships between DON and measures of Fusarium head blight intensity; and $P=$ probability value (significance level) for the analysis of variance (ANOVA)-like meta-analysis.

${ }^{\text {e }}$ Mean back-transformed correlation coefficients $(\bar{r})$ and lower $\left(C I_{\mathrm{L}}\right)$ and upper $\left(C I_{\mathrm{U}}\right)$ limits of the $95 \%$ confidence interval obtained through back-transformation of the corresponding transformed values. 
was a slight decline in the effect size with increase in the corresponding mean measure of disease intensity for disease index, incidence, diseased-head severity, and FDK.

\section{DISCUSSION}

Associations ranging from negative relationships to very strong positive relationships between Fusarium head blight intensity and DON content in harvested wheat grain have been reported $(1,2,4,7,12,13,24,25,27,31-34,45,47,51,54)$. This has led to many questions regarding the existence, or strength, of such a relationship and contrasting conclusions regarding the usefulness of disease intensity as an indirect indicator of DON $(2,15,34,45)$. Some studies reported that Fusarium head blight incidence (proportion of diseased spikes) was more strongly related with DON than were other measures of disease, whereas other studies reported that disease index (mean proportion of diseased spikelets per spike) was strongly related with DON $(2,31,32)$. Through a quantitative synthesis of the findings from published and unpublished studies using meta-analysis of transformed correlations, we observed that overall there were significant, positive relationships between all commonly used measures of Fusarium head blight intensity and DON.
Among the measures of disease quantified in the field, disease index had significantly higher correlation with DON than either incidence or diseased-head severity based on the paired differences between the transformed correlation coefficients (Table 2). The latter two measures of intensity had very similar correlations with DON. Whereas incidence measures the proportion of visibly diseased spikes in a sample, irrespective of how much of each individual spike is colonized, diseased-head severity measures the extent to which the average diseased spike is colonized, in terms of the proportion of spikelets (especially chaff tissue) with visible symptoms. On the basis of the known relationship between fungal biomass and DON $(7,36,51)$, more heavily colonized plant tissue is likely to have a greater fungal biomass, and consequently, higher DON content than the less colonized tissue. However, standard determination of DON at harvest $(14,15)$ and the method used in the studies analyzed herein, is based on a bulked sample of several ground-up seeds likely originating from several different spikes. Moreover, only some of the seeds from diseased spikes would be infected in the bulked sample, unless incidence and diseased-head severity were both $100 \%$ in the sample. Thus, measured DON in an assay typically is a composite value for seeds with different levels of DON (including those with $0 \mathrm{ppm}$ ) and different levels of fungal colonization. Since disease index ("plot

TABLE 4. Fisher's transformed and back-transformed correlation coefficients and corresponding statistics for the effect of wheat type on the relationship between different measures of Fusarium head blight intensity and deoxynivalenol accumulation in harvested wheat grain

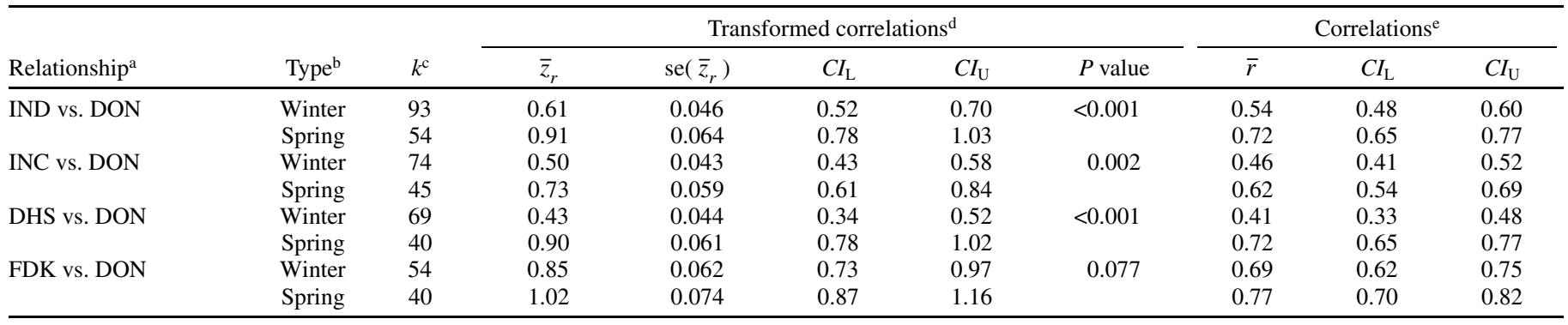

${ }^{a}$ DON = deoxynivalenol concentration in harvested grain $(\mathrm{ppm}) ; \mathrm{IND}=$ Fusarium head blight index (mean proportion of diseased spikelets per spike); INC $=$ Fusarium head blight incidence (proportion of diseased spikes); DHS = diseased-head severity ("conditional severity"; mean proportion of diseased spikelets per diseased spike); FDK = Fusarium-damaged kernels (proportion of visibly scabby kernels).

${ }^{\mathrm{b}}$ Data obtained from studies conducted using winter wheat and spring wheat cultivars.

c Total number of studies used in each analysis (based in the number of studies in which the indicated variable was measured).

d $\bar{z}_{r}=$ Mean Fisher's transformed correlation coefficient; se $\left(\bar{z}_{r}\right)=$ standard error of $\bar{z}_{r}$; lower $\left(C I_{\mathrm{L}}\right)$ and upper $\left(C I_{\mathrm{U}}\right)$ limits of the $95 \%$ confidence interval for $\bar{z}_{r}$; $\mathrm{Z}$ (standard normal) statistic from the analysis of relationships between DON and measures of Fusarium head blight intensity; and $P=$ probability value (significance level) for the analysis of variance (ANOVA)-like meta-analysis.

${ }^{\text {e }}$ Mean back-transformed correlation coefficients $(\bar{r})$ and lower $\left(C I_{\mathrm{L}}\right)$ and upper $\left(C I_{\mathrm{U}}\right)$ limits of the $95 \%$ confidence interval obtained through back-transformation of the corresponding transformed values.

TABLE 5. Fisher's transformed and back-transformed correlation coefficients and corresponding statistics for the effect of study type on the relationship between different measures of Fusarium head blight intensity and deoxynivalenol accumulation in harvested wheat grain

\begin{tabular}{|c|c|c|c|c|c|c|c|c|c|c|}
\hline \multirow[b]{2}{*}{ Relationship $^{\mathrm{a}}$} & \multirow[b]{2}{*}{ Study ${ }^{b}$} & \multirow[b]{2}{*}{$k^{\mathrm{c}}$} & \multicolumn{5}{|c|}{ Transformed correlations $^{\mathrm{d}}$} & \multicolumn{3}{|c|}{ Correlations $^{\mathrm{e}}$} \\
\hline & & & $\bar{z}_{r}$ & $\operatorname{se}\left(\bar{z}_{r}\right)$ & $C I_{\mathrm{L}}$ & $C I_{\mathrm{U}}$ & $P$ value & $\bar{r}$ & $C I_{\mathrm{L}}$ & $C I_{\mathrm{U}}$ \\
\hline IND vs. DON & Fungicide & 56 & 0.92 & 0.085 & 0.75 & 1.09 & 0.006 & 0.73 & 0.64 & 0.80 \\
\hline INC vs. DON & Genotype & 70 & 0.53 & 0.040 & 0.45 & 0.61 & & 0.49 & 0.42 & 0.54 \\
\hline \multirow[t]{2}{*}{ DHS vs. DON } & Fungicide & 34 & 0.71 & 0.102 & 0.51 & 0.91 & 0.267 & 0.61 & 0.47 & 0.72 \\
\hline & Genotype & 71 & 0.58 & 0.047 & 0.49 & 0.67 & & 0.52 & 0.45 & 0.58 \\
\hline
\end{tabular}

${ }^{\mathrm{a}} \mathrm{DON}=$ deoxynivalenol concentration in harvested grain $(\mathrm{ppm})$; IND = Fusarium head blight index (mean proportion of diseased spikelets per spike); INC = Fusarium head blight incidence (proportion of diseased spikes); DHS = diseased-head severity ("conditional severity"; mean proportion of diseased spikelets per diseased spike); FDK = Fusarium-damaged kernels (proportion of visibly scabby kernels).

${ }^{\mathrm{b}}$ Data obtained from fungicide trials and genotype evaluation studies.

${ }^{c}$ Total number of studies used in each analysis (based in the number of studies in which the indicated variable was measured).

d $\bar{z}_{r}=$ Mean Fisher-transformed correlation coefficient; se $\left(\bar{z}_{r}\right)=$ standard error of $\bar{z}_{r}$ lower $\left(C I_{\mathrm{L}}\right)$ and upper $\left(C I_{\mathrm{U}}\right)$ limits of the $95 \%$ confidence interval for $\bar{z}_{r} ; Z$ (standard normal) statistic from the analysis of relationships between DON and measures of Fusarium head blight intensity; and $P=$ probability value (significance level) for the ANOVA-like meta-analysis.

${ }^{\text {e }}$ Mean back-transformed correlation coefficients $(\bar{r})$ and lower $\left(C I_{\mathrm{L}}\right)$ and upper $\left(C I_{\mathrm{U}}\right)$ limits of the $95 \%$ confidence interval obtained through back-transformation of the corresponding transformed values. 
severity"; 48) is the product of incidence and diseased-head severity, IND $=$ DHS $\times$ INC (48), it represents the fraction of all spikelets with symptoms (including those that are disease free), or equivalently, the mean extent of fungal colonization for the entire sample at the assessment time. We would thus expect, in general, that disease index would have a higher association with DON than the more restrictive measures of disease intensity made in the field. This was indeed found in this analysis of 163 studies, although results for all three measures of disease intensity were similar.

Although disease index had a significantly overall higher correlation with DON than did either incidence or diseased-head severity, there were some individual studies in which the difference in correlation was close to 0, or in which either DHS or (especially) incidence had a higher correlation with DON than did index. Shaner and Buechley (45) also found that incidence had the highest correlation with DON in some studies in Indiana with winter wheat. Based on the meta-analysis results, these cases would be considered as simply the result of random variation operating at the study level. A higher association of incidence with DON could occur if total DON production in infected spikes was not directly dependent on the number of diseased spikelets (and hence seeds) because of some type of compensation mechanism. Since the meta-analysis gave no evidence that incidence had a higher association overall with DON than other measures of disease, such a hypothesis is not supported.

Given that the three measures of disease intensity are interrelated either statistically or mathematically, it is not surprising that similar distributions of correlations (or their transformation) were found. As indicated above, index is simply the product of DHS and INC. Moreover, index can be predicted from disease incidence using a nonlinear statistical model $(37,53)$. For practical reasons, incidence is the easiest measurement of intensity to ob- tain. From incidence, one can then predict index using equation 1 in Paul et al. (37), and if needed for some purposes, one can then estimate diseased-head severity from IND/INC. If one has the resources to directly measure index, then this is advisable since there is variation in index values around the predicted index value at a given level of disease incidence $(37,53)$. In many situations, however, it will not be affordable or practical to directly measure index, especially in surveys for disease intensity.

FDK, quantified after harvest, had the strongest association with DON, with a mean correlation coefficient that was significantly higher $(P<0.001)$ than all other correlation coefficients. The relatively weaker relationship between disease quantified in the field and DON may have been due to two main factors: the physical separation between the tissue assessed for disease and the tissue assessed for DON and the temporal separation between disease quantification and DON assessment. Visual assessments of disease were usually made at Feekes GS 11.2, based on the proportion of the spike (mainly chaff tissue) diseased, while DON was quantified after harvest as the amount of DON per unit weight of a bulked sample of ground (mostly chaff-free) kernels. Between the chaff and the kernel, there are several physical barriers that could potentially limit the movement of the fungus (and consequently DON) from the point of initial infection (on the glumes or chaff) to the kernel. Moreover, the growth of the fungus and the production of DON are highly weather dependent $(20,22)$, and unless conditions are favorable, both may be restricted to one part of the spikelet, leading to disparity between what is in the chaff and what reaches the kernel. Wang and Miller (51) and Miller et al. (36) reported substantially higher levels of DON in the chaff than in the kernel of wheat. The correlation between DON in the chaff and disease index $(r=0.85)$ was higher than the correlation between DON in the kernel and disease index $(r=$ 0.69) (51). Although a high proportion of diseased chaff tissue

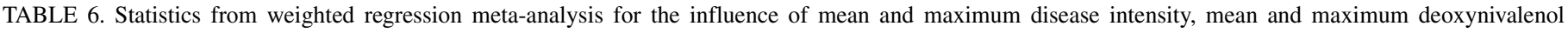

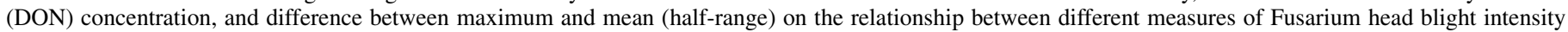
and deoxynivalenol accumulation in harvested wheat grain

\begin{tabular}{|c|c|c|c|c|c|c|c|c|}
\hline \multicolumn{2}{|c|}{ Relationship ${ }^{\mathrm{a}}$} & \multirow[b]{2}{*}{$k^{\mathrm{b}}$} & \multicolumn{6}{|c|}{ Statistics $^{\mathrm{c}}$} \\
\hline Response & Predictor & & $\hat{\alpha}$ & $\operatorname{se}(\hat{\alpha})$ & $\hat{\beta}$ & $\operatorname{se}(\hat{\beta})$ & $P$ & $R^{2}$ \\
\hline \multirow{6}{*}{$z_{r(\mathrm{IND})}$} & $\bar{x}_{\mathrm{IND}}$ & 147 & 0.843 & 0.067 & -0.005 & 0.002 & 0.020 & 0.033 \\
\hline & $\bar{x}_{\mathrm{DON}}$ & 147 & 0.725 & 0.056 & -0.001 & 0.004 & 0.819 & 0.0003 \\
\hline & $\operatorname{Max}_{\mathrm{IND}}$ & 146 & 0.806 & 0.081 & -0.002 & 0.001 & 0.177 & 0.011 \\
\hline & $\operatorname{Max}_{\mathrm{DON}}$ & 146 & 0.669 & 0.060 & 0.002 & 0.002 & 0.354 & 0.005 \\
\hline & $R n g_{\mathrm{IND}}$ & 146 & 0.712 & 0.073 & -0.00004 & 0.002 & 0.988 & 0.000 \\
\hline & $R n g_{\mathrm{DON}}$ & 146 & 0.643 & 0.057 & 0.005 & 0.003 & 0.109 & 0.016 \\
\hline \multirow{6}{*}{$z_{r(\mathrm{INC})}$} & $\bar{x}_{\mathrm{INC}}$ & 119 & 0.842 & 0.093 & -0.004 & 0.001 & 0.002 & 0.078 \\
\hline & $\bar{x}_{\mathrm{DON}}$ & 119 & 0.711 & 0.053 & -0.014 & 0.004 & 0.001 & 0.091 \\
\hline & $\operatorname{Max}_{\mathrm{INC}}$ & 119 & 1.002 & 0.140 & -0.005 & 0.002 & 0.002 & 0.079 \\
\hline & $\operatorname{Max}_{\mathrm{DON}}$ & 119 & 0.685 & 0.055 & -0.005 & 0.002 & 0.012 & 0.054 \\
\hline & $R n g_{\mathrm{INC}}$ & 119 & 0.548 & 0.064 & 0.001 & 0.002 & 0.536 & 0.003 \\
\hline & $R n g_{\mathrm{DON}}$ & 119 & 0.645 & 0.052 & -0.005 & 0.003 & 0.089 & 0.025 \\
\hline \multirow[t]{6}{*}{$z_{r(\mathrm{DHS})}$} & $\bar{x}_{\mathrm{DHS}}$ & 109 & 0.758 & 0.086 & -0.005 & 0.002 & 0.026 & 0.045 \\
\hline & $\bar{x}_{\mathrm{DON}}$ & 109 & 0.575 & 0.064 & 0.002 & 0.005 & 0.740 & 0.001 \\
\hline & $\operatorname{Max}_{\mathrm{DHS}}$ & 109 & 0.654 & 0.101 & -0.001 & 0.002 & 0.489 & 0.005 \\
\hline & $\operatorname{Max}_{\mathrm{DON}}$ & 109 & 0.488 & 0.063 & 0.004 & 0.002 & 0.035 & 0.040 \\
\hline & $R n g_{\mathrm{DHS}}$ & 109 & 0.504 & 0.080 & 0.003 & 0.003 & 0.210 & 0.015 \\
\hline & $R n g_{\mathrm{DON}}$ & 109 & 0.460 & 0.058 & 0.009 & 0.003 & 0.002 & 0.078 \\
\hline \multirow[t]{6}{*}{$z_{r(\mathrm{FDK})}$} & $\bar{x}_{\mathrm{FDK}}$ & 94 & 1.056 & 0.069 & -0.007 & 0.002 & 0.006 & 0.070 \\
\hline & $\bar{x}_{\mathrm{DON}}$ & 94 & 0.999 & 0.073 & -0.008 & 0.006 & 0.148 & 0.021 \\
\hline & $\operatorname{Max}_{\mathrm{FDK}}$ & 93 & 1.000 & 0.091 & -0.002 & 0.002 & 0.232 & 0.015 \\
\hline & $\operatorname{Max}_{\mathrm{DON}}$ & 93 & 0.922 & 0.079 & -0.001 & 0.003 & 0.840 & 0.0004 \\
\hline & $R n g_{\mathrm{FDK}}$ & 93 & 0.861 & 0.082 & 0.002 & 0.003 & 0.477 & 0.005 \\
\hline & $R n g_{\mathrm{DON}}$ & 93 & 0.877 & 0.074 & 0.002 & 0.004 & 0.574 & 0.003 \\
\hline
\end{tabular}

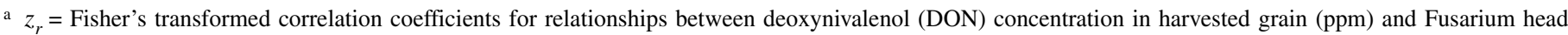
blight index (IND = mean proportion of diseased spikelets per spike), incidence (INC $=$ proportion of diseased spikes), diseased-head severity $(\mathrm{DHS}=$ mean proportion of diseased spikelets per diseased spike), and Fusarium-damaged kernels (FDK = proportion of visibly scabby kernels), $\bar{x}=$ arithmetic mean, Max $=$ maximum, and Rng = difference between maximum and mean (half-range).

b Total number of studies used in each analysis (based in the number of studies in which the indicated variable was measured).

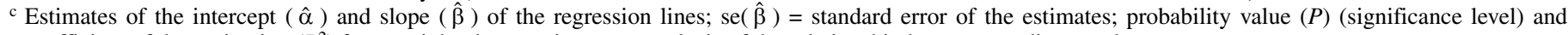
coefficient of determination $\left(R^{2}\right)$ from weighted regression meta-analysis of the relationship between predictor and response. 
would be expected to increase the probability of the fungus and DON reaching the grain, it does not necessarily translate into a proportionally high DON accumulation in the grain in a given study.

Depending on the study location and weather conditions, there may have been as much as 30 to 45 days between the time disease was assessed and the time of harvest followed by the analysis for DON. During that time, DON concentration may have increased at differential rates in the different studies, affecting the relationship between DON sampled at harvest and disease assessed at Feekes GS 11.2. Moreover, DON may accumulate even when no symptoms are evident at the time of visual disease assessment (22). Studies on the relationship between fungal biomass and DON reveal a strong association between presence of the fungus and DON contamination $(7,36,51)$. The relationship between the presence of the fungus and visual symptoms of the disease is often not as strong $(7,22)$.

For all measures of disease, the correlation coefficients varied from one study to another and based on the test of homogeneity, each study estimated different correlation coefficients (effect sizes) rather than providing estimates of a single effect size. This may have been due to the inherent difference among the studies in terms of the genotypes planted, weather conditions, pathogen population, crop production, and disease management practices, as well as other unknown random effects. These possible factors served as the impetus for the consideration of moderator variables in an attempt to explain some of the between-study variability. All categorical moderator variables (study location, study type, and wheat type) had significant effects on the overall correlation between disease intensity and DON. Results were similar for the four correlations considered, which was expected since the distributions of correlation coefficients across the studies were similar. In fact, the moderator-variable analyses could have been done for just one of the correlations (e.g., $r_{\text {IND }}$ ), but we showed all four sets of results for completeness. The influence of study location on transformed correlation coefficients may have been partly due to differences in the pathogen populations between U.S. spring wheat region, U.S. winter wheat region, and other wheat-growing regions (Canada, Europe, and Africa); more specifically, differences in their ability to produce DON or some complex effects of environment on the relationship. Miller et al (36) reported that isolates of $F$. graminearum from the U.S. and Canada varied in their ability to produce DON.

Adding to the differential ability of the pathogen to produce DON, cultivars are known to differ in DON accumulation (Type III resistance), resistance to infection (Type I resistance), resistance to disease spread within the spike (Type II resistance), and kernel infection (Type IV resistance) (32). In most of the fungicide studies, a single cultivar was used and the variation in DON and disease intensity would have been mainly because of treatment (products applied or rate of application) effects in a given environment. In the genotype studies, however, a range of genotypes with different levels of Type I to Type IV resistance were used in each study. This potentially led to a range of DON levels for a similar level of disease, or a range of disease intensities for a similar level of DON. The consequence of this would be the weaker correlation between disease intensity and DON found for genotype studies compared with fungicide studies in our analyses. Differences in the magnitude and relative range of Type I to Type IV resistance among cultivars within wheat type may have also been responsible for the differences in effect sizes between studies with spring and winter wheat cultivars.

Differences in weather conditions among locations also may have been responsible for some of the differences in mean effect size estimates across studies. Mesterhazy (31) reported (based on data collected between 1990 and 2000 in Europe) a high correlation between the amounts of DON produced in wheat grain and the amount of rainfall during the grain-fill period. This led the author to hypothesize that for a given level of disease (index or FDK), the level of DON may vary considerably from epidemic to epidemic as a function of precipitation. Hooker et al. (19) reported a relationship between DON accumulation in mature wheat grain (at heading) and the number of days of rainfall $>5$ $\mathrm{mm}$. In the latter study, rainfall and air temperature during heading explained between 55 and $79 \%$ of the variability in DON levels. The interaction between grain moisture content and temperature also has been shown to have a significant effect on DON accumulation $(20,22)$.

Weather information was unavailable for each study, so means and maxima of DON and disease intensity were used as indirect measures of environmental favorability for disease and DON accumulation. However, these variables only explained a small proportion of the between-study variability in effect size. A negative relationship (slope of the regression line) generally was found between transformed correlations and mean (and/or maximum) disease intensity and DON, indicating that the strength of the correlations between measures of disease and DON depended on the level of disease or DON concentration. Comparatively higher correlations occurred at low disease intensity (presumably with unfavorable environmental conditions) than at high disease intensity (presumably with favorable environmental conditions). The results of Lemmens et al. (24) concurred with our results. They clearly demonstrated that the highest correlation between the mean area under the disease progress curve (AUDPC) for Fusarium head blight and mean DON content of wheat grain $(r=0.89)$ occurred at relatively low mean AUDPC (approximately 13) and the lowest correlation $(r=0.08)$ occurred at relatively high mean AUDPC (approximately 40).

Relative to the true (i.e., population) correlation coefficient, the estimated correlation coefficient can be reduced by several factors related to properties of the study (21), which often are out of the control of the investigator. Hunter and Schmidt (21) refer to these factors as "artifacts of study imperfections". In particular, measurement error and range restriction in the variables being analyzed can reduce the realized correlation in a study (21). Corrections can be made to the estimated correlation coefficient if the reliability of the measurements were known. For the Fusarium head blight studies, there is certainly imprecision and possibly some statistical bias in the estimate of DON in seed and in the estimates of disease intensity, although the magnitude of the error is generally unknown, especially for the standard field experiments considered here. We speculate that differences in degree of measurement error among the analyzed studies contributed to the heterogeneity in effect sizes.

Range restriction occurs in a study when the range of values of the analyzed variables is less than that occurring in the larger population of possible values (21). This could occur with Fusarium head blight when only low values of DON and/or disease intensity occur in a study. The positive relationship in transformed correlations (i.e., positive slope) and the half-range DON variable (e.g., maximum minus mean DON; see Table 6) is limited evidence that range restriction could be partly responsible for some of the heterogeneity in effect sizes. However, the half-range DON variable was only significant at $P<0.05$ for one of the four effect sizes $\left(z_{r(\mathrm{DHS})}\right)$. A direct correction for range restriction is possible based on the standard deviations of the variables analyzed in the study relative to agreed-upon reference standard deviations for the variables in the larger population of studies, although there is controversy regarding the use of correction factors in general (26). There are no reference standard deviation values yet for disease intensity or DON, so this approach was not pursued. However, the low $R^{2}$ values for the regression equations indicate that range restriction is not of major importance in the heterogeneity of effect sizes.

Related to range restriction is censoring of data, where true low values are measured (incorrectly) as zero. This could occur when 
the assay for DON is not sensitive enough for low concentrations in the ground seed (14). There are complicated corrections for this (38) in terms of correlations, but these require large numbers of data points for each study. Nevertheless, censoring was not an issue in this investigation because $97 \%$ of the studies analyzed contained no zero values of DON.

Although most of the studies referred to herein were conducted according to a standard protocol, there still was some inherent variation among studies. In spite of these variations, relatively strong relationships were found between disease intensity and DON accumulation across studies, increasing the confidence in the relationship. However, given that high levels of precision and accuracy are needed to ensure that DON concentration does not exceed critical levels (44) in harvested grain (and consequently, their by-products), the use of disease intensity (including at-harvest measures such as FDK) as sole indicators of DON would not be recommended based on the meta-analytic results. Squaring the mean back-transformed correlation coefficients for each relationship between DON and disease one gets an estimate of the coefficient of determination for each relationship $\left(R^{2}\right)$, which can be used as indicator of how well DON could be predicted from disease. The overall $R^{2}$ value for relationships between index and DON, incidence and DON, diseased-head severity and DON, and Fusarium-damaged kernels and DON were $0.38,0.27,0.28$, and 0.53 , respectively, based on the mean correlations in Table 1 . This suggests that between 27 and $53 \%$ of the variation in DON content is explained, on average, by variation in disease intensity. From the results presented herein, it is clear that a large proportion of the variability remains unexplained (even after adjusting for the effects of moderator variables), suggesting that other unknown, unmeasured, or unmeasurable factors in typical field environments influence the relationship between DON and disease. Further analyses are needed to determine how DON accumulation changes with changing Fusarium head blight intensity, that is, the slope of the best-fitted regression line relating DON to disease intensity.

\section{ACKNOWLEDGMENTS}

Salaries and research support were provided by state and federal funds, especially the United States Wheat and Barley Scab Initiative, to the Ohio Agricultural Research and Development Center, The Ohio State University. We would like to thank D. Hershman of the University of Kentucky, C. Sneller of The Ohio State University, G. Milus of the University of Arkansas, and M. McMullen of North Dakota state University for providing data from uniform fungicide trials and Fusarium head blight screening nurseries.

\section{LITERATURE CITED}

1. Arseniuk, E., Foremska, E., Goral, T., Chelkowski, J. 1999. Fusarium head blight reactions and accumulation of deoxynivalenol (DON) and some of its derivatives in kernels of wheat, triticale and rye. J. Phytopathol. 147:577-590.

2. Bai, G.-H., Plattner, R., Desjardins, A., and Kolb, F. 2001. Resistance to Fusarium head blight and deoxynivalenol in wheat. Plant Breed. 120:1-6.

3. Bai, G.-H., and Shaner, G. 1994. Scab of wheat: Prospects for control. Plant Dis. 78:760-766.

4. Champeil, A., Fourbet, J. F., Dore, T., and Rossignol, L. 2004. Influence of cropping system on Fusarium head blight and mycotoxin levels in winter wheat. Crop Prot. 23:531-537.

5. Desjardins, A. E., Proctor, R. H., Bai, G., McCormick, S. P., Shaner, G., Buechley, G., and Hohn, T. M. 1996. Reduced virulence of trichothecenenonproducing mutants of Gibberella zeae in wheat field tests. Mol. PlantMicrobe Interact. 9:775-781.

6. Dunn, O. J., and Clark, V. 1969. Correlation coefficients measured on the same individuals. J. Am. Stat. Assoc. 64:366-377.

7. Edwards, S. G., Pirgozliev, S. R., Hare, M. C., and Jenkinson, P. 2001. Quantification of trichothecene-producing Fusarium Species in harvested grain by competitive PCR to determine efficacies of fungicides against Fusarium head blight of winter wheat. Appl. Environ. Microbiol. 67:1575-1580.
8. Field, A. P. 2001. Meta-analysis of correlation coefficients: A monte carlo comparison of fixed- and random-effects methods. Psychol. Methods 6:161-180.

9. Fisher, R. A. 1928. Statistical Methods for Research Workers. 2nd ed. Oliver and Boyd, London.

10. Glass, G. 1976. Primary, secondary, and meta-analysis of research. Educ. Sci. 5:3-8.

11. Gurevitch, J. and Hedges, L. V. 1993. Meta-analysis: Combining the results of independent experiments. Pages 378-425 in: Design and Analysis of Ecological Experiments. S. M. Scheiner and J. Gurevitch, eds. Chapman and Hall, London.

12. Haidukowski, M., Pascale, M., Perrone, G., Pancaldi, D., Campagna, C., and Visconti, A. 2005. Effect of fungicides on the development of Fusarium head blight, yield and deoxynivalenol accumulation in wheat inoculated under field conditions with Fusarium graminearum and Fusarium culmorum. J. Sci. Food Agric. 85:191-198.

13. Hall, M. D., and Van Sanford, D. A. 2003. Diallel analysis of Fusarium head blight resistance in soft red winter wheat. Crop Sci. 43:1663-1670.

14. Hart, L. P., and Schabenberger, O. 1998. Variability of vomitoxin in truckloads of wheat in a wheat scab epidemic year. Plant Dis. 82:625-630.

15. Hart, P., and Schabenberger, O. 2001. Early detection of deoxynivalenol in wheat grain. Pages 164-167 in: Proc. 2001 Natl. Fusarium Head Blight Forum, Erlanger, KY.

16. Hedges, L.V., and Olkin, I. 1980. Vote-Counting methods in research synthesis. Psychol. Bull. 88: 359-369.

17. Hedges, L. V. and Vevea, J. L. 1998. Fixed- and random-effects models in meta-analysis. Psychol. Methods 3:486-504.

18. Hittner, J. B., May, K., and Silver, N. Clayton. 2003. A monte carlo evaluation of tests for comparing dependent correlations. J. Gen. Psychol. 130:149-168.

19. Hooker, D. C., Schaafsma, A. W., and Tamburic-Ilincic, L. 2002. Using weather variables pre- and post-heading to predict deoxynivalenol content in winter wheat. Plant Dis. 86:611-619.

20. Hope, R., Aldred, D., and Magan, N. 2005. Comparison of environmental profiles for growth and deoxynivalenol production by Fusarium culmorum and F. graminearum on wheat grain. Lett. Appl. Microbiol. 40:295-300.

21. Hunter, J. E., and Schmidt, F. L. 1994. Correcting for sources of artificial variation across studies. Pages 323-338 in: The Handbook of Research Synthesis. H. Cooper and L. V. Hedges, eds. Russell Sage Foundation, New York.

22. Lacey, J., Bateman, G. L., and Mirocha, C. J. 1999. Effects of infection time and moisture on development of ear blight and deoxynivalenol production by Fusarium spp. in wheat. Ann. Appl. Biol. 134:277-283.

23. Large, E. C. 1954. Growth stages in cereals. Plant Pathol. 3:128-129.

24. Lemmens, M., Buerstmayr, H., Krska, R., Schuhmacher, R., Grausgruber, H., and Ruckenbauer, P. 2004. The effect of inoculation treatment and long-term application of moisture on Fusarium head blight symptoms and deoxynivalenol contamination in wheat grains. Eur. J. Plant Pathol. 110:299-308.

25. Lemmens, M., Haim, K., Lew, H., and Ruckenbauer, P. 2004. The effect of nitrogen fertilization on Fusarium head blight development and deoxynivalenol contamination in wheat. J. Phytopathol. 152:1-8.

26. Lipsey, M. W., and Wilson, D. B. 2001. Practical Meta-Analysis. Sage Publications Inc., Thousand Oaks, CA.

27. Liu, W., Langseth, W., Skinnes, H., Oleif, E. N., and Sundheim, L. 1997. Comparison of visual head blight ratings, seed infection levels, and deoxynivalenol production for assessment of resistance in cereals inoculated with Fusarium culmorum. Eur. J. Plant Pathol. 103:589-595.

28. McCormick, S. 2003. The role of DON in pathogenicity. Pages 165-183 in: Fusarium Head Blight of Wheat and Barley. K. J. Leonard and W. R. Bushnell, eds. The American Phytopathology Society, St. Paul, MN.

29. McMullen, M., Jones, R., and Gallenburg, D. 1997. Scab of wheat and barley: A re-emerging disease of devastating impact. Plant Dis. 81:1340-1348.

30. McRoberts, N., Hughes, G., and Madden, L. V. 2003. The theoretical basis and practical application of relationships between different disease intensity measures in plants. Ann. Appl. Biol. 142:191-211.

31. Mesterhazy, A. 2002. Role of deoxynivalenol in aggressiveness of Fusarium graminearum and $F$. culmorum and in resistance to Fusarium head blight. Eur. J. Plant Pathol. 108:675-684.

32. Mesterhazy, A., Bartok, T., Mirocha, C. G., and Komoroczy, R. 1999. Nature of wheat resistance to Fusarium head blight and the role of deoxynivalenol for breeding. Plant Breed. 118:97-110.

33. Miedaner, T., Reinbrecht, C., Lauber, U., Schollenberger, M., and Geiger, H. H. 2001. Effects of genotype and genotype-environment interaction on deoxynivalenol accumulation and resistance to Fusarium head blight in rye, triticale, and wheat. Plant Breed. 120:97-105.

34. Miedaner, T., Schneider, B., and Geiger, H. H. 2003. Deoxynivalenol (DON) content and Fusarium head blight resistance in segregating populations of winter rye and winter wheat. Crop Sci. 43:519-526. 
35. Miller, J. D., Greenhalgh, R., Wang, Y. Z., and L, Ming. 1991. Trichothecene chemotype of three Fusarium species. Mycologia 83:121-130.

36. Miller, J. D., Young, J. C., and Sampson, D. R. 1985. Deoxynivalenol and Fusarium head blight resistance in spring cereals. Phytopathol. Z. 113:359-367.

37. Paul, P. A., El-Allaf, S. M., Lipps, P. E., and Madden, L. V. 2005. Relationships between incidence and severity of Fusarium head blight on winter wheat in Ohio. Phytopathology 95:1049-1060.

38. Piepho, H.-P., Thoni, H., and Muller, H.-M. 2002. Estimating the productmoment correlation in samples with censoring on both variables. Biom. J. 44:657-670.

39. Proctor, R. H., Hohn, T. M., and McCormick, S. P. 1995. Reduced virulence of Gibberella zeae caused by disruption of the trichothecene toxin biosynthesis gene. Mol. Plant-Microbe Interact. 8:593-601.

40. Proctor, R. H., Hohn, T. M., and McCormick, S. P. 1997. Restoration of wild-type virulence to tri5 disruption mutants of Gibberella zeae via gene reversion and mutant complementation. Microbiology 143:2583-2591.

41. Rosenberg, M. S., Garrett, K. A., Su, Z., and Bowden, R. L. 2004. Metaanalysis in plant pathology: Synthesizing research results. Phytopathology 94:1013-1017.

42. Rosenthal, R. 1979. The "file-drawer problem" and tolerance for null results. Psychol. Bull. 86:638-641.

43. Rosenthal, R. 1991. Meta-Analytic Procedures for Social Research. Rev. Ed. Sage, Newbury Park, CA.

44. Rotter, B. A., Prelusky, D. B., and Pestka, J. J. 1996. Toxicology of deoxynivalenol (vomitoxin). J. Toxicol. Environ. Health 48:1-34.

45. Shaner, G., and Buechley, G. 2004. Relation between head blight severity and DON in natural epidemics of FHB. Page 518 in: Proc. 2nd Int. Symp.
Fusarium Head Blight, Orlando, FL.

46. Snijders, C. H. A. 1990. Fusarium head blight and mycotoxin contamination of wheat, a review. Neth. J. Plant Pathol. 96:187-198.

47. Snijders, C. H. A., and Perkowski, J. 1990. Effect of head blight caused by Fusarium culmorum on toxin content and weight of wheat kernels. Phytopathology 80:566-570.

48. Stack, R. W., and McMullen, M. P. 1998. A visual scale to estimate severity of Fusarium head blight in wheat. NDSU Extension Service: Small Grains Publications. Online Publication/PP-1095.

49. Steiger, J. H. 1980. Tests for comparing elements of a correlation matrix. Psychol. Bull. 87:245-251.

50. Wang, M. C., and Bushman, B. J. 1999. Integrating Results through MetaAnalytic Review Using SAS software. SAS Institute, Cary, NC.

51. Wang, Y. Z., and Miller, J. D. 1988. Effects of Fusarium graminearum metabolites on wheat tissue in relation to Fusarium head blight resistance. J. Phytopathol. 122:118-125.

52. Windels, C. E. 1999. Economic and social impacts of Fusarium head blight: Changing farms and rural communities in the Northern Great Plains. Phytopathology 90:17-21.

53. Xu, X.-M., Parry, D. W., Edwards, S. G., Cooke, B. M., Doohan, F. M. Maanen, A., Brennan, J. M., Monaghan, S., Moretti, A., Tocco, G., Mule, G., Hornok, L., Giczey, G., Tatnell, J., Nicholson, P., and Ritieni, A. 2004. Relationship between the incidences of ear and spikelet infection of Fusarium ear blight in wheat. Eur. J. Plant Pathol. 110:959-971.

54. Zhou, W.-C., Kolb, F. L., Bai, G.-H., Domier, L. L., and Yao, J.-B. 2002. Effect of individual sumai 3 chromosomes on resistance to scab spread within spikes and deoxynivalenol accumulation within kernels in wheat. Hereditas 137:81-89. 